\title{
Prevalence of Male Genital Tuberculosis in Indian Infertile Couples and Its Correlation with Female Genital Tuberculosis
}

\author{
${ }^{1}$ Asha Baxi, ${ }^{2}$ Hansali Neema, ${ }^{3}$ Pooja Kadi, ${ }^{4}$ Dawal Baxi, ${ }^{5}$ Manila Kaushal
}

\begin{abstract}
Objectives: To study the prevalence of male and female genital tuberculosis in Indian infertile couples as detected by semen polymerase chain reaction (PCR) and endometrial PCR and to study the correlation of semen TB PCR with endometrial TB PCR of sexual partner.
\end{abstract}

Design: Noncomparative retrospective observational series.

Patients: A total of 100 couples presenting with infertility at clinic were enrolled after obtaining informed consent.

Interventions: All couples were tested for mycobacterium tuberculosis (MTB) by nested PCR on the semen and the endometrial sample. The results of PCR of sexual partners were mutually correlated.

Results: Out of 108 couples, 56 males and 52 females had positive PCR in the sample of semen and the endometrium. Also, 30 couples simultaneously had both semen PCR and endometrial PCR positive.

Conclusion: Addition of PCR tests to the routine clinical and laboratory assessments may add to the detection of subclinical urogenital infection and the timely evaluation of the asymptomatic partner of the infected person can improve the early detection of a silent but potentially devastating infection.

Keywords: Infertility, Male genital tuberculosis, Semen TB PCR.

How to cite this article: Baxi $A$, Neema $H$, Kadi $P$, Baxi $D$, Kaushal M. Prevalence of Male Genital Tuberculosis in Indian Infertile Couples and Its Correlation with Female Genital Tuberculosis. J South Asian Feder Obst Gynae 2016;8(1):13-15.

\section{Source of support: Nil}

Conflict of interest: None

Date of received: 12 December 2015

Date of acceptance: 18 February 2016

Date of publication: March 2016

\section{INTRODUCTION}

India is home to over 3.4 million tuberculosis (TB) patients-about one-fifth of the global figure-making itthe most TB prevalent country. ${ }^{1}$ The estimated incidence

\footnotetext{
${ }^{1}$ Director, ${ }^{2,5}$ Consultant, ${ }^{3,4}$ Clinical Assistant

${ }^{1-5}$ Department of Obstetrics and Gynecology, Disha Fertility and Surgical Center, Indore, Madhya Pradesh, India

Corresponding Author: Asha Baxi, Director, Department of Obstetrics and Gynecology, Disha Fertility and Surgical Center Indore, Madhya Pradesh, India, e-mail: aabaxi@gmail.com
}

of TB in India is 1.96 million new cases annually with 0.8 million new smear positive cases annually and 75 new smear positive PTB cases/1 lakh population per year. The estimated mortality due to TB is 330,000 deaths each year averaging over 1000 deaths a day and 2 deaths every 3 minutes. The prevalence of TB infection in India is estimated to be $40 \%$ ( $~ 400$ million with a $10 \%$ lifetime risk of TB disease in the absence of HIV). ${ }^{2}$

The incidence of TB is increasing worldwide, with more than $20 \%$ of cases exhibiting extrapulmonary manifestations. The genitourinary tract is the most common site of extrapulmonary TB. Genital TB is usually secondary to pulmonary TB and usually presents with atypical manifestations. Only 20 to $30 \%$ of patients with genitourinary TB have a history of pulmonary infection. Tuberculosis of the male and female reproductive tract can result in infertility. Infertility may be the first and the only presentation of genitourinary TB and patients may have no recollection of any other symptoms. ${ }^{6}$ Considering the high endemicity of genital TB in India and its strong association with infertility, this study is done to evaluate the prevalence of genital TB in Indian infertile population and to observe the mutual correlation between male and female genital TB.

\section{AIMS AND OBJECTIVES}

- To study the prevalence of male and female genital TB in Indian infertile couples as detected by semen polymerase chain reaction (PCR) and endometrial PCR.

- To study the correlation of semen TB PCR with endometrial TB PCR of sexual partner.

\section{MATERIALS AND METHODS}

This was a retrospective study carried out at Disha Fertility and Surgical Center, Indore, over a period of 1 year from August 2008 to July 2009. Couples presenting with infertility were evaluated. Thorough menstrual, obstetric, sexual, occupational, medical and history was taken as per predesigned proforma. Physical examination and basic investigations for infertility were done.

Semen analysis was carried out and analyzed according to World Health Organization (WHO) criteria. Semen sample was subjected to Nested PCR for Mycobacterium 
tuberculosis (MTB) complex. All samples were sent to the same lab for analysis. All females were tested for MTB by Nested PCR on the endometrial sample. The results of PCR of sexual partners were mutually correlated.

\section{RESULTS}

This study observes and correlates the endometrial TB PCR results with semen TB PCR results in a large group comprising a total of 200 men and women presenting as infertile couple. It was observed that out of 108 couples, 56 males and 52 females had positive PCR in the sample of semen and the endometrium (Table 1). Also, 30 couples simultaneously had both semen PCR and endometrial PCR positive. Total 52 women had positive endometrial PCR. Twenty-two women had positive endometrial PCR with negative semen PCR of their partner. Also, 26 males had positive semen PCR with negative endometrial PCR of their sexual partner (Table 2).

\section{DISCUSSION}

Tuberculosis can affect virtually any organ system in the body and can be devastating if undiagnosed or untreated. The increasing prevalence of TB in both immunocompetent and immunocompromised individuals in recent years makes this disease a topic of universal concern. Also, the link between TB and HIV is quiet significant with $\mathrm{WHO}$ estimating that $5 \%$ of $\mathrm{TB}$ patients are also coinfected with HIV. ${ }^{1}$

The actual prevalence of infection in the Indian community is still not known exactly and is probably underestimated. This can be attributed mainly to the nonspecific clinical features, poor diagnostic tools for confirmation of the disease, absence of a standardized system for notification of the disease, population explosion with a large population under poverty line with malnutrition and high vulnerability to TB, lack of awareness in the community for seeking early and complete treatment, associated social stigma with the disease and high rate of coinfection with HIV.

The infection can involve any part of the male and female reproductive tract. It may affect the testis, epididymis, vas deferens, seminal vesicles, prostate and

Table 1: Prevalence of semen TB PCR in males

\begin{tabular}{lll}
\hline Semen TB PCR & Detected & Not detected \\
\hline No. of patients & 56 & 44 \\
\hline
\end{tabular}

Table 2: Prevalence of positive semen TB PCR and endometrial TB PCR among couple

\begin{tabular}{llll}
\hline Semen TB PCR & Detected & Not detected & Total \\
\hline TB PCR detected & 30 & 22 & 52 \\
Not detected & 26 & 22 & 48 \\
\hline Total & 56 & 44 & 100 \\
\hline
\end{tabular}

the ejaculatory ducts. Infertility usually results from the inflammation and scarring that follow the infection, resulting in distortion of the normal anatomy and causing obstruction.

Moreover, this silent invader of genital tract tends to create diagnostic dilemma because of varied clinical presentations, diverse results on imaging and laparoscopy and limitations of histopathological, serological, bacteriological and culture methods. Culture methods, which have been considered gold standard in proving genital TB, fail to exclude mycobacterial infection. Furthermore, Mycobacterium is a temperamental bacillus that needs 4 to 5 weeks to show growth on LJ media and 2 weeks time on Radiometric BACTEC media. ${ }^{4}$ The minimum mycobacterial concentration at which histopathological evidence appears is $10,000 \mathrm{bacilli} / \mathrm{ml}$ and for positive culture the required concentration is 1000 bacilli/ml and 10 to $100 \mathrm{bacilli} / \mathrm{ml}$ for LJ media and BACTEC media respectively. Polymerase chain reaction has been able to detect TB bacilli in the concentration as low as $10 \mathrm{bacilli} / \mathrm{ml} .{ }^{5}$ Hence, in combination with accurate clinical assessment PCR test seems to be a very promising diagnostic tool.

Based on the current knowledge of vast prevalence of TB and the high sensitivity and specificity of PCR as a diagnostic tool for $\mathrm{TB}$, present study is undertaken. Moreover, due to high endemicity and the characteristic tendency of the bacilli of silent invasion, there is a high potential of mutual transmission of infection between the asymptomatic sexual partners particularly from males to females. The most common mode of genital tract involvement is usually hematogenous in both males and females but ascending mode via semen is also an established mode of transmission. Patients with subclinical infection can potentially transmit Mycobacterium to their sexual partner via semen or direct contact in a manner similar to HIV, syphilis and other STD.

It was observed in the present study that $56 \%$ of males and $52 \%$ of females had positive PCR in the sample of semen and the endometrium suggesting a very high prevalence in the infertile population. Also, $30 \%$ of couples had both male and female genital TB simultaneously. The present observation suggests that there is a possibility that Mycobacterium subclinically resides in the male or female genital tract and gets sexually transmitted particularly from males to females via seminal fluid and may contribute to both male and female factor infertility simultaneously. An important aspect of genital infections in male may be that male accessory sex glands may act as reservoirs for the organism, increasing the possibility of transmission to the females.

It is well-established that early detection and treatment is vital to prevent structural and functional damage to 
genital tract which is usually in the form of fibrosis. Considering the $30 \%$ rate of simultaneous infection among couples and the likelihood of mutual transmission of infection, timely evaluation of the asymptomatic partner of the infected person can improve the early detection of such a potentially devastating infection. Total 52 women had positive endometrial PCR. Twenty-two women had positive endometrial PCR with negative semen PCR of their partner. Also, 26 males had positive semen PCR with negative endometrial PCR of their sexual partner. In such cases, institution of timely treatment cannot only cure the disease but can also prevent the potential transmission to the unaffected partner.

The WHO report on the TB in Southeast Asia in $2008^{1}$ and epidemiological survey by Chakraborty ${ }^{3}$ has estimated $40 \%$ prevalence in general population in India. The relatively higher prevalence in the present study can be partly explained by the high risk group of infertile patients selected as the study group. Since, the accessibility of the sample of semen and endometrium had practical implications, the study group was confined to the infertile couples for the present study. Further studies the same field involving a larger group of general population can provide statistically more precise prevalence rates. Presently, the literature search has revealed scanty data to correlate the observations related to possibility of mutual transmission among sexual partners.

It is essential to emphasize that available studies in the literature do show a strong association of genital $\mathrm{TB}$ with both male and female infertility but there is limited data available comparing the potential of mutual transmission between the couples. Probably, this has been one encouraging factor for us to undertake present study on a large group comprising of 200 patients.

The presence of MTB PCR was considered as a diagnostic tool for genital TB for the present study. As the mycobacterium is a known obligatory pathogen of genital tract, presence of its DNA was considered as genital tract infection which may be subclinical, latent or clinical. Although MTB PCR has a known sensitivity and specificity of $>90 \%$ in body fluids like sputum and urine, its efficacy in semen still needs to be established and this may be considered as a possible limitation of present study. Further research in the same field involving general population and a larger group with therapeutic trials can provide new insights in the field of TB and infertility.

To conclude, the observations of the present study suggests that considering the high endemicity of genital TB, presentation with infertility alone should be considered as high-risk group for TB. Since the disease requires prompt diagnosis and definitive treatment, addition of PCR tests to the routine clinical and laboratory assessments may add to the detection of subclinical urogenital infection. Also, once one of the partner is diagnosed with genital TB, other partner should be evaluated for the infection due to high probability of mutual transmission so that microbial cure and better fertility outcomes can be offered to the asymptomatic couples seeking treatment for infertility.

\section{REFERENCES}

1. Tuberculosis in the South-East Asia Region. The Regional Report: World Health Organization; 2008.

2. Gopi PG, Subramani R, Santha T, Chandrasekaran V, Kolappan C, Selvakumar N, Narayanan PR. Estimation of burden of tuberculosis in India for the year 2000. Indian J Med Res 2005 Sep;122(3):243-248.

3. Chakraborty AK. Epidemiology of tuberculosis: current status in India. Indian J Med Res 2004 Oct;120(4):248-276.

4. Ketch VM. Newer diagnostic techniques for TB. Indian J Med Res 2004;120:418-428.

5. Bhanu NV, Singh UB, Chakraborty M, Suresh N, Arora J, Rana T, Takkar D, Seth P. Improved diagnostic value of PCR in the diagnosis of female genital tuberculosis leading to infertility. J Med Microbiol 2005;54:927-931.

6. Kumar R. Reproductive tract tuberculosis and male infertility. Indian J Urol 2008;24:392-395. 